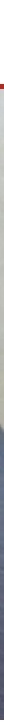

(c) Aus dem Beitrag von A. Janssen in dieser Ausgabe

\title{
Das komplexe regionale Schmerzsyndrom (CRPS)
}

\author{
Psyche, Körper und Traditionelle Chinesische Therapie (TCM)
}

Das Schwerpunktthema dieser Ausgabe befasst sich mit dem komplexen regionalen Schmerzsyndrom oder kurz CRPS (abgekürzt von der englischen Bezeichnung Complex Regional Pain Syndrom). Dieser Begriff wurde 1999 von der International Association for the Study of Pain (IASP) eingeführt und sollte eigentlich alle Begriffe, die bis dato zur Beschreibung dieses Symptomenkomplexes verwendet wurden, ablösen. Vor allem im deutschen Sprachraum wird aber weiterhin oft der Begriff des Morbus Sudeck verwendet, der auf den deutschen Erstbeschreiber Paul Sudeck zurückgeht.

Das CRPS ist keine seltene Erkrankung. Trotzdem wird es in der klinischen Praxis oft nicht erkannt, so dass der Zeitabstand von Beschwerdebeginn bis zur Diagnosestellung mehrere Monate betragen und dadurch die Prognose des Patienten ungünstig beeinflussen kann. Wann an ein CRPS zu denken ist und welche wesentlichen Punkte bei Diagnostik und

Deutsche Zeitschrift für Akupunktur 2020 • 63 (2): 83-84

https://doi.org/10.1007/s42212-020-00257-5

๑) Springer Medizin Verlag GmbH, ein Teil von

Springer Nature 2020
Therapie gemäß den aktuellen Leitlinien zu beachten sind, hat für Sie Herr Dr. Andreas Böger zusammengefasst. Herr Dr. Böger ist der Chefarzt der Schmerzmedizin, Manuelle Therapie und Naturheilverfahren der DRK-Kliniken Nordhessen und Regionalleiter der Deutschen Gesellschaft für Schmerzmedizin (DGS). Die Klinik hat sich in den letzten 10 Jahren $\mathrm{zu}$ einem Schwerpunktzentrum für $\mathrm{Pa}$ tienten mit CRPS entwickelt. So liegt bei knapp 10 \% der rund 4000 in den 3 Sektoren ambulant/tagesklinisch/stationär behandelten Patienten die Diagnose CRPS vor. Die CRPS-Patienten werden entweder ambulant oder im Rahmen des Kasseler Aktiv Programms (KAP) stationär multimodal behandelt. Auch Akupunktur wird dort zur Behandlung eingesetzt und Herr Dr. Böger präsentiert Ihnen, zusätzlich zu seiner umfassenden Übersicht, ein Fallbeispiel zur Akupunktur bei CRPS.

Da beim CRPS auch viel über eine psychische Beteiligung diskutiert wird, sogar bis hin zu Thesen über eine „CRPS-Persönlichkeit“, war es uns wichtig, Ihnen einen guten Überblick zu diesem komplexen Thema zu bieten. Wir freuen uns sehr, dass wir dafür Frau Dr. rer. biol. hum. Dipl. Psych. Heike Schulte-Göcking gewinnen konnten. Sie arbeitet seit Jahren als Psychologin in der Interdiszi- plinären Schmerzambulanz des Klinikums der Ludwig-Maximilians-Universität München Großhadern und hat über das Thema Körperschemastörungen und Psyche beim CRPS promoviert. Für dieses Schwerpunktheft hat sie wichtige Erkenntnisse aus ihrer Forschung und ihrer klinischen Erfahrung für Sie zusammengefasst.

Hinsichtlich der Möglichkeiten von TCM und Akupunktur beim CRPS präsentieren wir Ihnen zwei spannende Anwendungsbeispiele: Frau Dr. Barbara Schneider, Leiterin der schmerztherapeutischen Abteilung des DRK-Krankenhauses Altenkirch-Hachenburg berichtet über einen spannenden Fall zur Kombination von TCM-Wissen mit dem naturheilkundlichen Einsatz von Blutegeln. Frau Dr. Anabelle Janssen von der Abteilung für Rehabilitation der BG-Klinik Murnau stellt Ihnen die Spiegelakupunktur vor - eine innovative Akupunkturtechnik vor, die auf der Spiegeltherapie beruht und eine elegante Lösung darstellt, die direkte Nadelung der betroffenen Extremität zu vermeiden. Der Spiegeltherapie wird eine modulierenden Wirkung auf die kortikale Plastizität zugeschrieben. Dies könnte auch als pathophysiologisches Erklärungsmodell für einen möglichen Akupunktureffekt diskutiert 
werden. Dieser Einfluss der Akupunktur auf die kortikalen Repräsentationszonen konnte bereits in Grundlagenuntersuchungen mittels funktioneller MRT bei Karpaltunnelsyndrom festgestellt werden [1-3]. Bei CRPS-Patienten wurde diese Untersuchung bislang nicht durchgeführt, jedoch gilt die kortikale Plastizität als ein bekanntes und viel beschriebenes Phänomen beim CRPS, das auch als eine der potenziellen Pathomechanismen diskutiert wird [4-6]. Diese Beobachtungen sind der Grund dafür, dass einige physikalische Therapien, wie beispielsweise die Spiegeltherapie oder das Training der sensorischen Diskriminierung, sich primär auf die Wiederherstellung der sensomotorischen Repräsentation der betroffenen Extremität konzentrieren. Aus diesen Überlegungen und den beschriebenen klinischen Beobachtungen könnte man schlussfolgern, dass positive Akupunktureffekte beim CRPS über einen regulierenden Einfluss auf die kortikale Plastizität zustande kommen. Allerdings ist die wissenschaftliche Datenlage zum Effekt der Akupunktur bis dato noch sehr unzureichend:

In PubMed finden sich zu den Begriffen „CRPS“ und „acupuncture“ lediglich anekdotische Fallberichte [7-9] sowie eine randomisiert kontrollierte Studie (RCT) an insgesamt 14 Teilnehmern, die keinen signifikanten Unterschied zwischen Sham- und Verumakupunktur zeigen konnte [10]. Dies ist ernüchternd, insbesondere da kaum Therapien beim CRPS ein gutes Evidenzlevel aufweisen können. Sogar die frühere Goldstandardtherapie mit Sympathikusblockaden war mehr aufgrund pathophysiologischer Überlegungen empfohlen worden als evidenzbasiert [11] und wird aufgrund der Datenlage heute auch nicht mehr standardmäßig empfohlen. Die Empfehlungen zur Bisphosphonattherapie in der aktuellen S1-Leitlinie [12] beruhen auf insgesamt 5 RCTs zu verschiedenen Präparaten, teilweise mit sehr kleinen Fallzahlen ( $n=10$, 13, 32, 40 und 82). Auch die Empfehlung zur Steroidstoßtherapie beim akuten CRPS beruht auf 2 RCTs, einmal mit 13 und einmal mit 60 Teilnehmern. Angesichts der schwachen Evidenzlage vermeintlicher Standardtherapien kann eine Anwendung der Akupunktur aufgrund ihrer nachgewiesenen analgetischen Wirkung im Einzelfall und im Rahmen eines multimodalen Behandlungskonzeptes trotz der fehlenden Evidenz im Rahmen einer Risiko-Nutzen-Abwägung in Betracht gezogen werden. Da die Therapie im schmerzfreien Raum stattfinden soll, ist mit lokaler Nadelung sehr zurückhaltend umzugehen. Vielmehr sind Fernpunkte, Mikrosysteme und Kontralateraltechniken indiziert, eine interessante lokale Stimulationstechnik könnte die Laserakupunktur aufgrund ihrer nachgewiesenen analgetischen, antiödematösen und regenerativen Wirkungen sein.

\section{Literatur}

1. Napadow Vet al (2007) Hypothalamus and amygdala response to acupuncture stimuli in Carpal Tunnel Syndrome. Pain 130(3):254-266

2. Napadow Vet al (2006) Somatosensory cortical plasticity in carpal tunnel syndrome - a cross-sectional fMRl evaluation. Neuroimage 31(2):520-530

3. Napadow Vet al (2007) Somatosensory cortical plasticity in carpal tunnel syndrome treated by acupuncture. Hum Brain Mapp 28(3):159-171

4. Maihofner Cet al (2003) Patterns of cortical reorganization in complex regional pain syndrome. Neurology 61(12):1707-1715

5. Maihofner Cet al (2004) Cortical reorganization during recovery from complex regional pain syndrome. Neurology 63(4):693-701

6. Marinus J et al (2011) Clinical features and pathophysiology of complex regional pain syndrome. Lancet Neurol 10(7):637-648

7. Hommer DH (2012) Chinese scalp acupuncture relieves pain and restores function in complex regional pain syndrome. Mil Med 177(10):1231-1234

8. Kim JM et al (2014) Bee venom pharmacopuncture: an effective treatment for complex regional pain syndrome. J Pharmacopuncture 17(4):66-69

9. Sprague M, Chang JC (2011) Integrative approach focusing on acupuncture in the treatment of chronic complex regional pain syndrome. J Altern Complement Med 17(1):67-70

10. Korpan Mlet al (1999) Acupuncture in the treatment of posttraumatic pain syndrome. Acta Orthop Belg 65(2):197-201

11. O'Connell NE et al (2016) Local anaesthetic sympathetic blockade for complex regional pain syndrome. Cochrane Database Syst Rev. https:// doi.org/10.1002/14651858.CD004598.pub4

12. Deutsche Gesellschaft für Neurologie (2018) Diagnostik und Therapie komplexer regionaler Schmerzsyndrome (CRPS), S1-Leitlinie. Leitlinien für Diagnostik und Therapie in der Neurologie.

\section{Korrespondenzadresse}

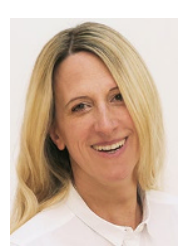

Dr. Sybille Kramer

Klinik für Orthopädie,

Physikalische Medizin und

Rehabilitation,

Klinikum der Universität

München

Marchioninistr. 15, 81377 München, Deutschland Sybille.Kramer@med.uni-muenchen.de

Interessenkonflikt. S. Kramer gibt an, dass kein Interessenkonflikt besteht. 